\title{
Soliton tunneling with sub-barrier kinetic energies
}

\author{
J. A. González \\ Centro de Física, Instituto Venezolano de Investigaciones \\ Científicas, Apartado Postal 21827, Caracas 1020-A, Venezuela \\ A. Bellorín \\ Departamento de Física, Facultad de Ciencias, Universidad Central de \\ Venezuela, Apartado Postal 47588, Caracas 1041-A, Venezuela \\ L. E. Guerrero \\ Departamento de Física, Universidad Simón Bolívar, Apartado 89000, \\ Caracas 1080-A, Venezuela
}

(July 6, 2018)

\begin{abstract}
We investigate (theoretically and numerically) the dynamics of a soliton moving in an asymmetrical potential well with a finite barrier. For large values of the width of the well, the width of the barrier and/or the height of the barrier, the soliton behaves classically. On the other hand, we obtain the conditions for the existence of soliton tunneling with sub-barrier kinetic energies. We apply these results to the study of soliton propagation in disordered systems.
\end{abstract}

05.45. Yv, 52.35.Mw, 73.40.Gk

The escaping process of a particle from a potential well, as that shown in Fig. 1 (barrier crossing), is a problem of great importance in almost all areas of physics [1].

In the case of a classical particle, this escape should occur over the barrier with the help of external perturbations (e.g., thermally activated barrier crossing) [1]. On the other hand, a quantum particle can perform tunneling with certain probability $p<1$.

In the present letter we address the question: what happens if, in the potential well, instead of a point-like particle we have a soliton? This is very relevant to Skyrmion models for nucleon physics, the motion of fluxons in long Josephson junctions with impurities, the dynamics of domain walls in ferroelectric materials in the presence of inhomogeneous electric fields, and many other physical systems where the solitons move in a potential created by inhomogeneities and external forces 2 2. 5.

It is well-known that a soliton can behave as a classical particle in some physical systems [2,6]. However, recently there has been a great interest in non-classical behaviors of the soliton [7 [14]. In particular, we are interested in extremely surprising phenomena that can occur when the soliton behaves as an extended object [8 10].

Among these phenomena is the soliton tunneling suggested by Kälbermann in a beautiful paper [15. However, this was a numerical work and the tunneling with sub-barrier kinetic energies was not observed in the specific situations in which the numerical experiments were performed.

In the present letter we show (theoretically and numerically) that the tunneling with sub-barrier kinetic energies is, indeed, possible!

As an example we consider the perturbed $\phi^{4}$-equation:

$$
\phi_{t t}-\phi_{x x}-\frac{1}{2}\left(\phi-\phi^{3}\right)=F(x) \text {. }
$$


The external force $F(x)$ is such that a point-like soliton would feel an effective potential like that shown in Fig. 1.

When the soliton is treated as a point-like particle, the zeroes of $F(x)$ are equilibrium points [9]. The zeroes $x_{0}$ $\left(F\left(x_{0}\right)=0\right)$ for which $\left[\frac{d F(x)}{d x}\right]_{x=x_{0}}>0$ are stable equilibrium positions. In the opposite case, they are unstable.

For our theoretical calculations we will use the force $F(x)$ defined in the following way:

$$
\begin{gathered}
F(x)=F_{1}(x), \text { for } x<x^{*}, \\
F(x)=c, \text { for } x>x^{*},
\end{gathered}
$$

where $F_{1}(x)=\frac{1}{2} A\left(A^{2}-1\right) \tanh (B x)+\frac{1}{2} A\left(4 B^{2}-\right.$ $\left.A^{2}\right) \frac{\sinh (B x)}{\cosh ^{3}(B x)}, x^{*}\left(x^{*}>0\right)$ is the point where $F_{1}(x)$ has a local minimum $\left(\frac{d F\left(x^{*}\right)}{d x}=0\right)$, and $c=F_{1}\left(x^{*}\right)$.

The condition $|F(-\infty)|=\frac{1}{2} A\left|A^{2}-1\right|<\frac{1}{3 \sqrt{3}}$ should hold for the stability of the soliton as a whole. This force allows us to solve the problem of soliton dynamics in a neighborhood of the equilibrium points [8]10]. For instance, the stability problem $\left(\phi(x, t)=\phi_{k}(x)+f(x) e^{\lambda t}\right)$ of the equilibrium point $x_{0}=0$ is reduced to the eigenvalue problem $\hat{L} f=\Gamma f$, where $\hat{L}=-\partial_{x}^{2}+$ $\left(\frac{3}{2} A^{2}-\frac{1}{2}-\frac{3 A^{2}}{2 \cosh ^{2}(B x)}\right)$ and $\Gamma=-\lambda^{2}$. The eigenvalues of the discrete spectrum are given by $\Gamma_{n}=-\frac{1}{2}+B^{2}(\Lambda+$ $\left.2 \Lambda n-n^{2}\right)$ where $\Lambda(\Lambda+1)=\frac{3 A^{2}}{2 B^{2}}$.

Our analysis reveals that if $A^{2}>1$ and $4 B^{2}<1$, the force given by Eqs. (2)-(3) possesses the desired properties, i.e. there is a zero that would correspond to a stable equilibrium position in a point $x=-d(d>0)$ and a zero in the point $x=0$ that would correspond to an unstable equilibrium position and would serve as a potential barrier. For $x>0$ the potential is a monotonically decreasing function.

In fact, if $2 B^{2}\left(3 A^{2}-1\right)<1$, then the soliton behaves classically. In this case, the soliton feels the barrier in the point $x=0$. If the soliton is situated in a vicinity of point $x=0$ with zero initial velocity and with the center of mass in a point $x<0$, it will not move to the right of point $x=0$.

On the other hand, if $2 B^{2}\left(3 A^{2}-1\right)>1$, the soliton will move to the right, crossing the barrier even if its center of mass is placed in the minimum of the potential and its initial velocity is zero (see Fig. 2). In this case the soliton performs tunneling with sub-barrier kinetic energy!

We should remark that this phenomenon is possible only when the distance $d$ between the minimum of the potential well and the maximum of the potential barrier holds the inequality $d<2.17$, where $d=$ $\left(\frac{1}{B}\right) \operatorname{arccosh}\left(\sqrt{\frac{A^{2}-4 B^{2}}{A^{2}-1}}\right)$. This can be interpreted in the sense that the wavelength of the soliton should be comparable with the width of the potential well. 
In this context, the work 14] addresses the differences and similarities of soliton phenomena with those of point particles. Although the studied phenomena are very different, we can say that the main conclusion is in consistency with our previous papers [8 10] and the present one: the soliton behaves as a particle only when the width of the potential makes the soliton appears as point-like. Otherwise, the soliton can have wave-like extended character.

The condition for soliton tunneling can be written in more physical terms. In fact, the force $F(x)$ can be defined by the value $F_{0}=\left|\lim _{x \rightarrow-\infty} F(x)\right|$, the width of the barrier $S$ and the local maximum $F_{m}$ of the force between the points $x=-d$ and $x=0$ (see inset in Fig. 1). In these terms, the approximate condition for the existence of soliton tunneling is $F_{0}>\frac{F_{m} S^{2}}{6}$. This inequality shows that greater values of $F_{0}$ support the tunneling, while greater values of $F_{m}$ and $S$ can thwart the tunneling.

We have performed numerical experiments with the force $F(x)$ defined as in Eqs. (2)-(3) and with many other functions which produce an effective potential as that shown in Fig. 1. We have been able to control the values of $F_{0}, F_{m}$ and $S$. Figure 3 shows the numerical experiments. The dots on the curve separates two zones: the zone in which the soliton tunneling is possible (upper zone) and the zone in which the soliton tunneling is impossible (lower zone). Note that the relation $F_{0}=\frac{F_{m} S^{2}}{6}$ is approximately satisfied. With other forces, the results are qualitatively equivalent.

Sometimes, it is convenient to see the condition for the existence of soliton tunneling in terms of a parameter that defines the potential. An important characteristic of the potential is the height of the potential barrier: $V_{m}$. Figure 4 shows the relationship between $F_{0}$ and $V_{m}$ while the bifurcation condition $2 B^{2}\left(3 A^{2}-1\right)=1$ holds. For points above the curve, the tunneling occurs. For points under the curve, the soliton behaves classically. That is, as was expected, the height of the potential barrier is an opposing factor with respect to the tunneling.

Note that the values of the potential $V(x)$ (or the force $F(x))$ for $x \ll-d$ can influence the tunneling. This is in contrast with the behavior of a point-like classical particle.

We should remark directly that, in this phenomenon, there is no violation of the energy conservation law. In fact, the energy of the whole system

$$
\begin{array}{r}
E \equiv \int_{-\infty}^{\infty}\left[\frac{1}{2}\left(\frac{\partial \phi}{\partial t}\right)^{2}+\frac{1}{2}\left(\frac{\partial \phi}{\partial x}\right)^{2}-\frac{1}{4} \phi^{2}+\frac{1}{8} \phi^{4}\right. \\
-F(x) \phi+c] d x
\end{array}
$$

is conserved. In this case, the soliton does not behave as a classical point-like particle. The soliton, as an extended object, possesses a wave-mechanical behavior. Even when the center of mass is situated in a point of minimal potential energy and with zero kinetic energy, the system as a whole can have enough energy to make 
the soliton able to cross the barrier (although the center of mass goes through the barrier). Kälbermann's example of a high jumper is felicitous [15]. However, we should notice the presence of nonlocal effects during this process.

The propagation of solitons in disordered media has been studied intensively in last years [16]. There is consensus in the conclusion that nonlinearity can modify the effects of localization and the transmission is improved. Nevertheless, even in a nonlinear system supporting solitons, if the latter behave as point-like particles, they can be trapped in the zeroes of the force $F(x)$. We stress that the phenomenon of soliton tunneling can enhance even more the transmission.

Consider Eq. (11) with $F(x)$ defined in such a way that it possesses many zeroes, maxima and minima (see Fig. 5). This system describes an array of inhomogeneities. The array can be studied as a series of elements with two zeroes and a maximum. If for each element the condition $F_{0}>\frac{F_{m} S^{2}}{6}$ is satisfied, then the soliton can cross the whole inhomogeneous zone (we have checked this numerically). The array can be completely disordered. If the condition is fulfilled, there is no localization.

In many systems 16 20 the solitons play the role of means of transport: they can carry energy and/or charge. Our result shows that, when soliton tunneling is possible, the soliton can be a very efficient carrier.

Kälbermann 15] investigated impurities that are introduced in the Hamiltonian density in the following way:

$$
H=\frac{1}{2}\left(\frac{\partial \phi}{\partial t}\right)^{2}+\frac{1}{2}\left(\frac{\partial \phi}{\partial x}\right)^{2}+\frac{1}{8} p\left(\phi^{2}-1\right)^{2}
$$

where $p=p_{0}+U(x) ; p_{0}$ is a constant, $U(x)$ is the perturbation that describes the impurity.

The perturbations used in the numerical experiments in Ref. [15] are given by the function

$$
U(x)=\frac{h_{1}}{\cosh ^{2}\left(\frac{x-x_{1}}{a_{1}}\right)}+\frac{h_{2}}{\cosh ^{2}\left(\frac{x-x_{2}}{a_{2}}\right)} .
$$

This perturbation is equivalent to an effective potential with a maximum, a minimum or a combination of both. Out of the inhomogeneous zone, the potential tends to zero exponentially. Suppose that the soliton moves from the left with a kinetic energy less than the maximum of the potential barrier 15 . In this case the soliton tunneling does not exist. As we have shown, for the soliton tunneling (among other conditions) it is necessary to have a soliton moving in a potential well where $V(x)$ takes values (out of the potential well) greater than that of the barrier. Nevertheless, with localized impurities like the ones introduced in Eq. (5), the soliton tunneling can also be observed. This is possible with a perturbation $U(x)$ with the features shown in Fig. 6. Even in this case, if the soliton moves from the left (in zone A) with subbarrier kinetic energy, then the soliton tunneling does not occur. For the tunneling, the soliton should be placed in 
zone B. Of course, the rest of the conditions should be satisfied.

The sine-Gordon soliton usually is thought to be a very point-like object. In fact, the unperturbed sineGordon equation is integrable and its soliton solution does not have discrete, internal (shape) modes. Nevertheless, when the perturbations are not given by Dirac's $\delta$-functions, the sine-Gordon soliton also is able to excite a great number of shape modes 21] and behaves as an extended object.

Consider the perturbed sine-Gordon equation

$$
\phi_{t t}-\phi_{x x}+\sin \phi=F(x) \text {, }
$$

where

$$
F(x)= \begin{cases}-F_{0} & \text { for } x<x_{0}, \\ \frac{a}{\cosh ^{2}(b x)}-F_{0}^{*} & \text { for }-x_{0} \leq x \leq x_{0}, \\ -F_{0} & \text { for } x>x_{0}\end{cases}
$$

$x_{0}$ and $F_{0}^{*}$ are chosen such that the function $F(x)$ is continuous.

Let us see only two examples. Let $F_{0}=0.25$ and $b=$ 0.55 (fixed). For $a=0.45$, the soliton remains trapped in the potential well. It behaves classically. For $a=0.35$, the soliton escapes from the potential well, crossing the barrier. We should emphasize that in both cases the force $F(x)$ would correspond to a system with a potential well and a barrier if the soliton behaves classically.

We would like to remark that, in our study, the positioning of the soliton at a certain point is done considering the equation of motion. The initial configuration is always a solution of the equation of motion (we can use both the static and the time dependent solutions in dependence on the physical process that led to the given situation). That is, we never use an initial configuration which the soliton can not reach by any means. For example, let us explain a physical situation in which the soliton can be positioned inside the potential well depicted in Fig. 1. Suppose a soliton is captured by a localized inhomogeneity (there are many such situations discussed in the review paper [6] and the experimental papers quoted therein). Then, we apply an external constant force (e.g. for the Josephson fluxon [5] this is a dc bias current). In that case, the soliton can be placed in an effective potential similar to that shown in Fig. 1. If it had lost all its kinetic energy [22,6], then we can use the static soliton as an initial condition. In fact, we think that the conditions for soliton tunneling could have been satisfied in the experimental situation described in [22].

We conclude that the phenomenon of soliton tunneling is robust and generic. We believe this phenomenon can be observed also in other physical systems bearing solitons, topological defects, vortices, spiral waves, etc. 
[1] P. Hänggi, P. Talkner and M. Borkovec, Rev. Mod. Phys. 62, 251 (1990).

[2] A. R. Bishop, J. A. Krumhansl, and S. E. Trullinger, Physica D 1, 1 (1980), and references therein.

[3] T. H. R. Skyrme, Proc. R. Soc. London, Ser. A 260, 127 (1961).

[4] G. S. Adkins, C. R. Nappi, and G. Witten, Nucl. Phys. B 228, 552 (1983).

[5] D. W. McLaughlin and A. C. Scott, Phys. Rev. A 18, 1652 (1978); Solitons in Action, edited by K. Lonngren and A. Scott (Academic, New York, 1978).

[6] Y. S. Kivshar and B. A. Malomed, Rev. Mod. Phys. 61, 763 (1989).

[7] Y. S. Kivshar, Zhang Fei, and L. Vázquez, Phys. Rev. Lett. 67, 1117 (1991).

[8] J. A. González, B. A. Mello, L. I. Reyes, and L. E. Guerrero, Phys. Rev. Lett. 80, 1361 (1998).

[9] J. A. González and J. A. Hołyst, Phys. Rev. B 45, 10338 (1992).

[10] J. A. González and B. A. Mello, Phys. Scr. 54, 14 (1996).

[11] Y. S. Kivshar, D. E. Pelinovsky, T. Cretegny, and M. Peyrard, Phys. Rev. Lett. 80, 5032 (1998).

[12] D. K. Campbell, M. Peyrard, and P. Sodano, Physica D 190, 165 (1986).

[13] J. A. González, L. E. Guerrero, and A. Bellorín, Phys. Rev. E 54, 1265 (1996); B. A. Mello, J. A. González, L. E. Guerrero, and E. López-Atencio, Phys. Lett. A 244, 277 (1998).

[14] G. Kälbermann, Phys. Lett. A 252, 37 (1999).

[15] G. Kälbermann, Phys. Rev. E 55, R6360 (1997).

[16] S. A. Gredeskul and Y. S. Kivshar, Phys. Rep. 216, 1 (1992).

[17] A. J. Heeger, S. Kivelson, J. R. Schrieffer, and W.-P. Su, Rev. Mod. Phys. 60, 781 (1988).

[18] A. S Davidov, Solitons in Molecular Systems (Naukova Dumka, Kiev, 1984).

[19] M. A. Rice, Phys. Lett. A 71, 152 (1979); Phys. Lett. A 73, 153 (1979).

[20] Yu Lu, Solitons and Polarons in Conducting Polymers (World Scientific, Singapore, 1988).

[21] J. A. González, Mod. Phys. Lett. B 6, 1867 (1992).

[22] H. Akoh, S. Sakai, A. Yagi, and H. Hayakawa, IEEE Trans. Magn. 21, 737 (1985).

FIG. 1. Potential $V(x)$ for the soliton escaping problem. The inset shows the force $F(x)$. Note that in all figures the quantities plotted are dimensionless.

FIG. 2. Numerical simulation of the soliton tunneling with sub-barrier kinetic energy. The pale curve is the potential and the bold curve is the soliton. The inflexion point is approximately the center of mass. (a) $t=0, V(t=0)=0$; (b-f) show the dynamics in succesive time instants. 
FIG. 3. Conditions for the existence of soliton tunneling with sub-barrier kinetic energy. The curve $F_{0}=\frac{F_{m} S^{2}}{6}$ separates the upper zone, where the soliton tunneling is possible from the lower zone, where the soliton tunneling is impossible. The filled circles represent numerical experiments.

FIG. 4. Conditions for the existence of soliton tunneling with sub-barrier kinetic energy involving the parameters $F_{0}$ and $V_{m}$. The other parameters remain fixed.

FIG. 5. Disordered array of inhomogeneities. The soliton can move through the whole array.

FIG. 6. Combination of impurities for Eq. (5). The soliton tunneling with sub-barrier kinetic energy is possible from zone B to zone C. 


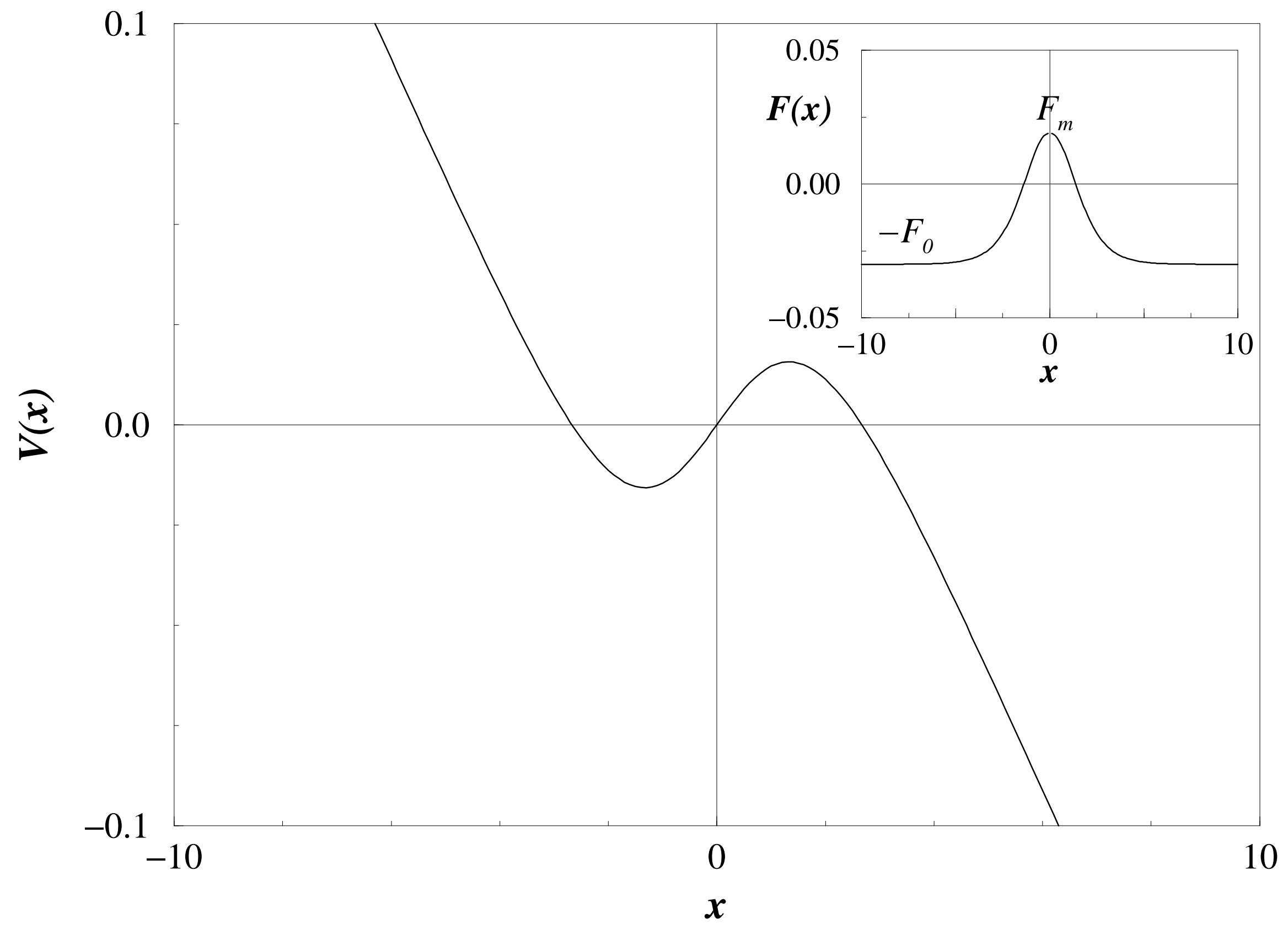




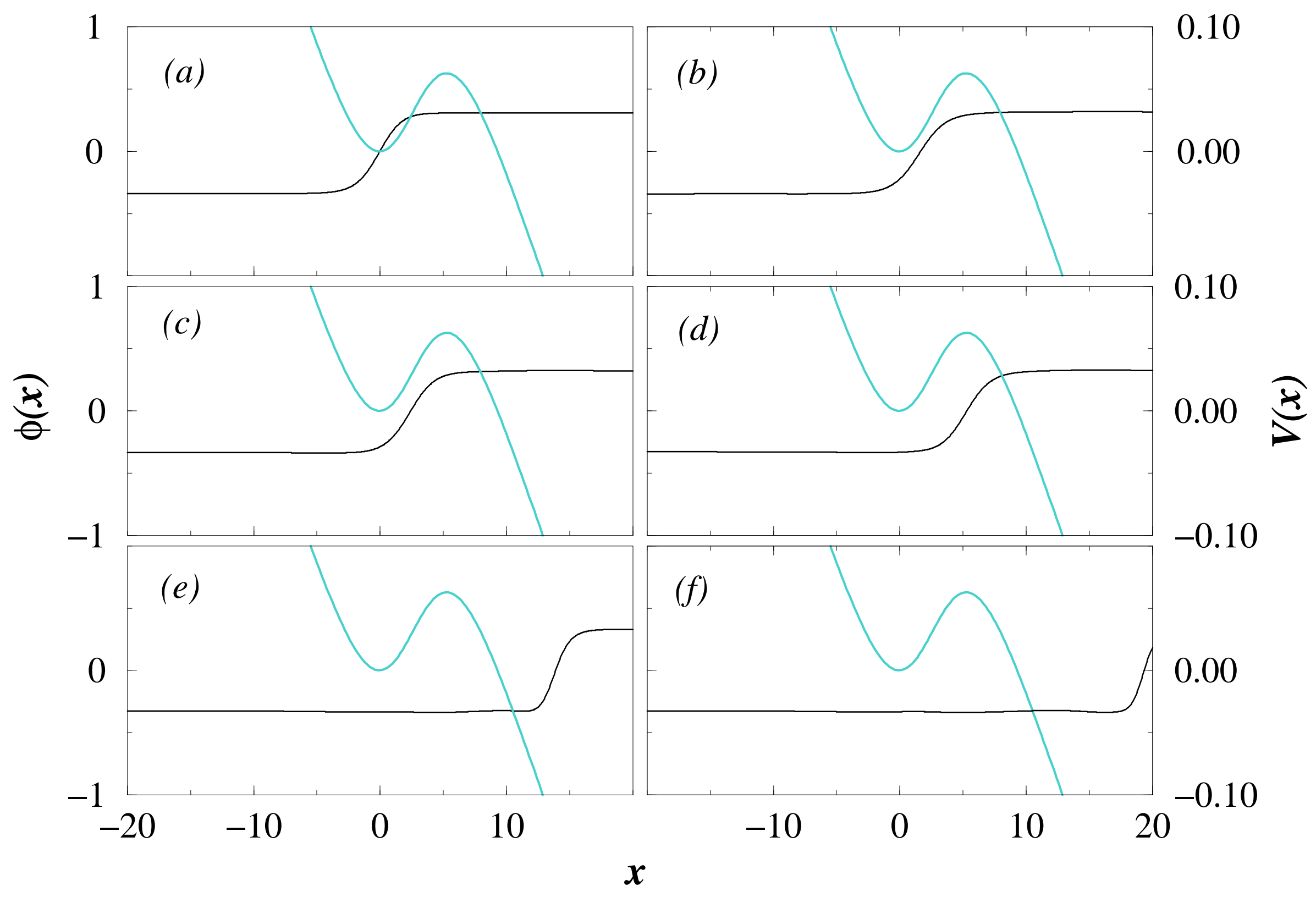




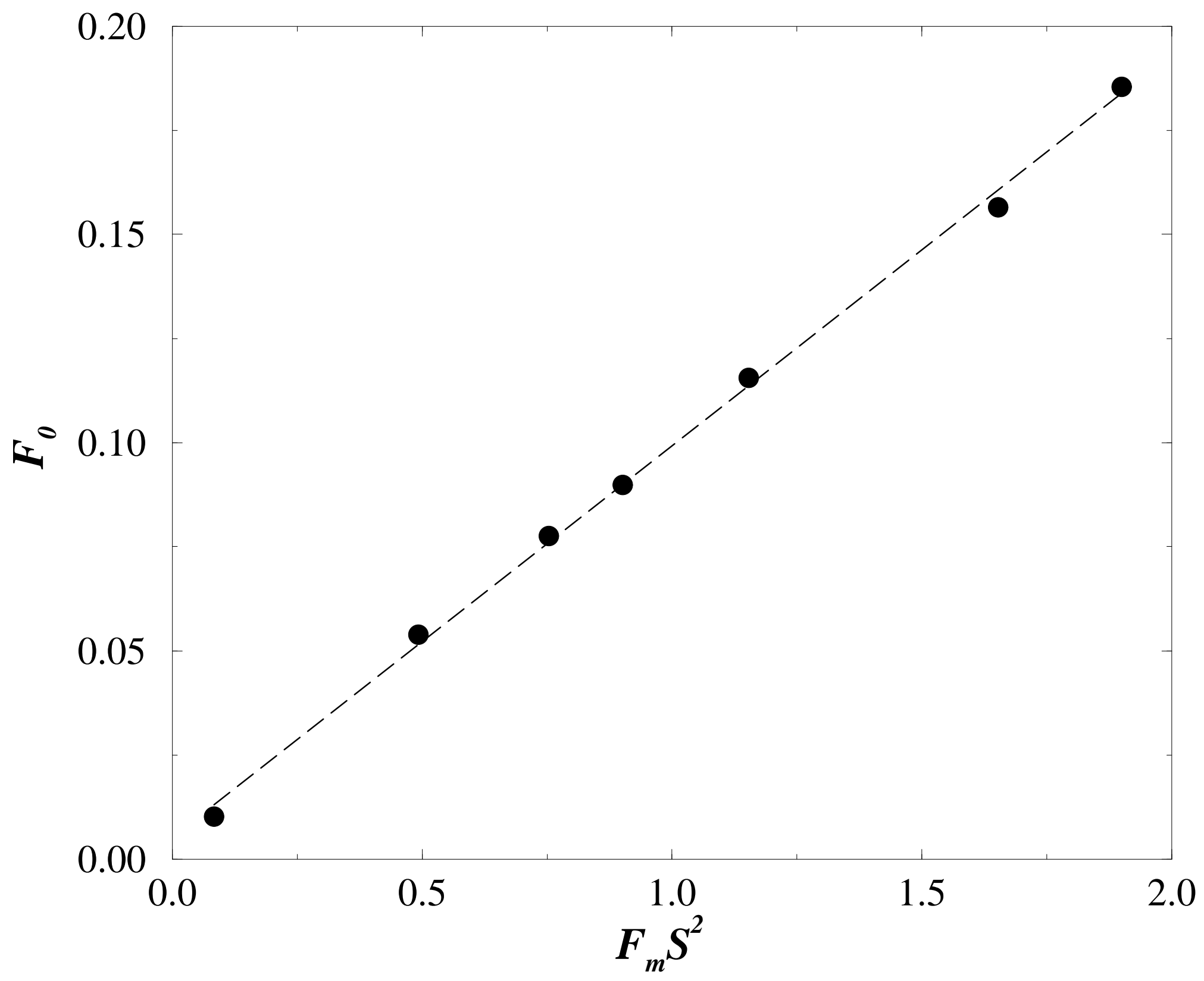




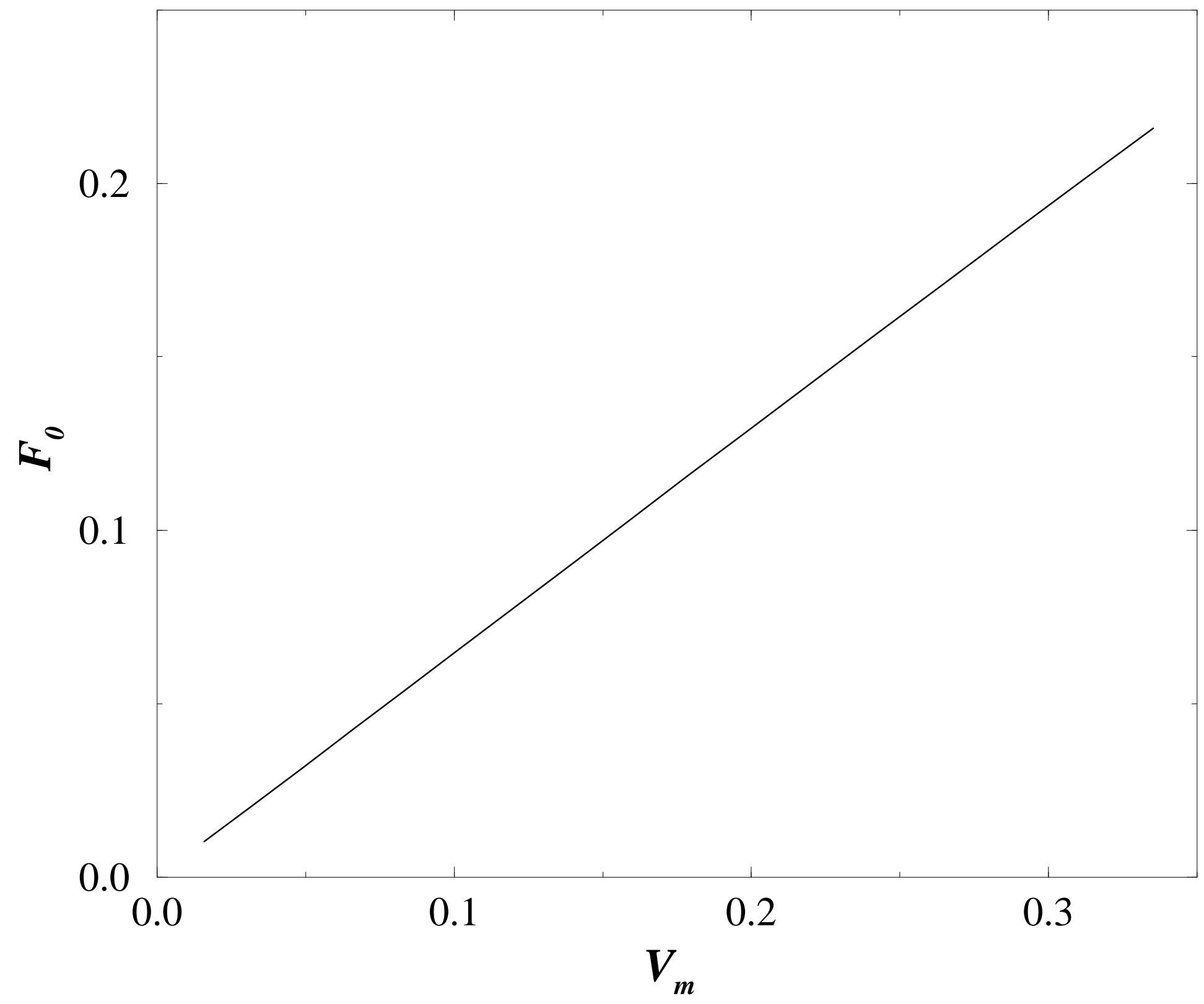




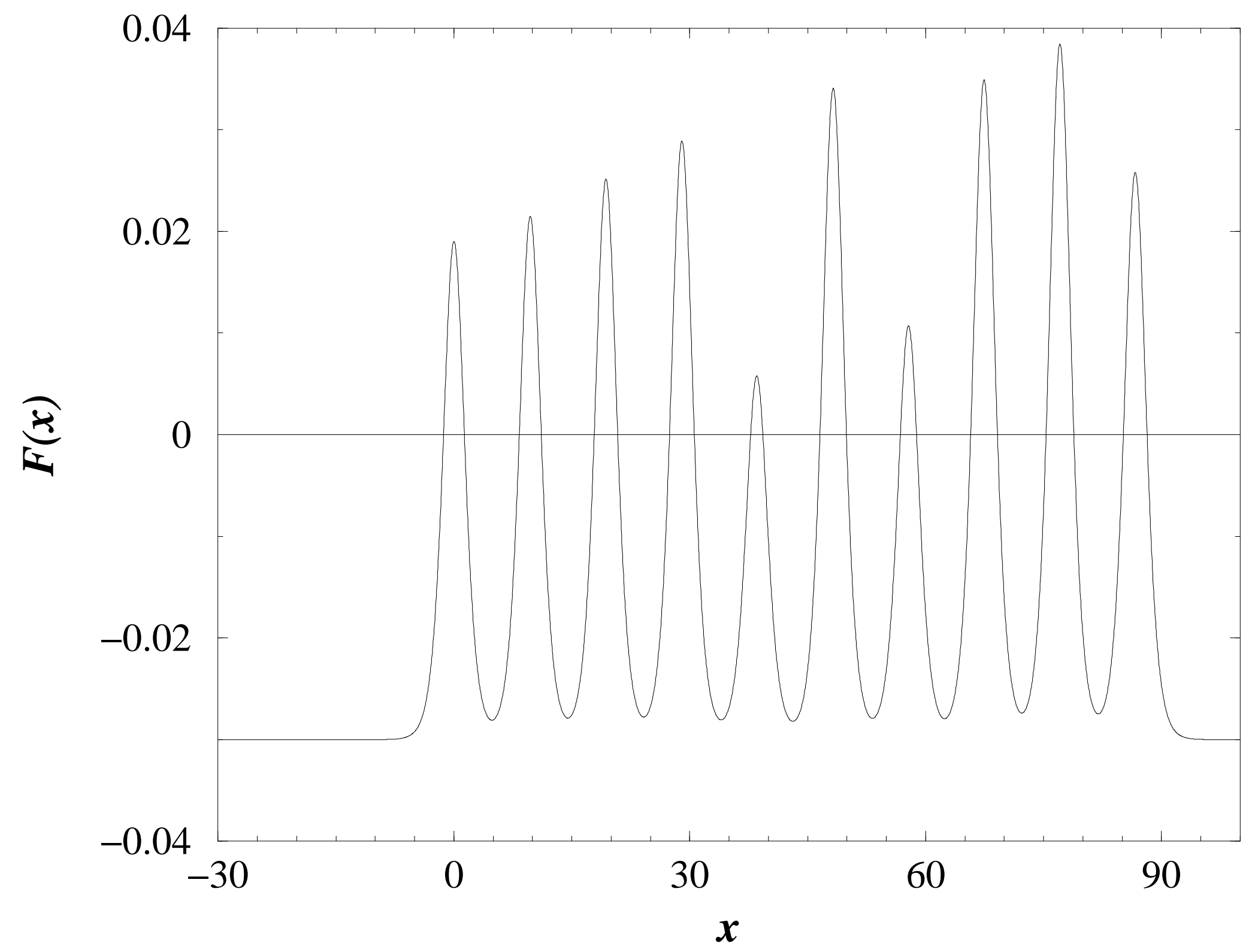




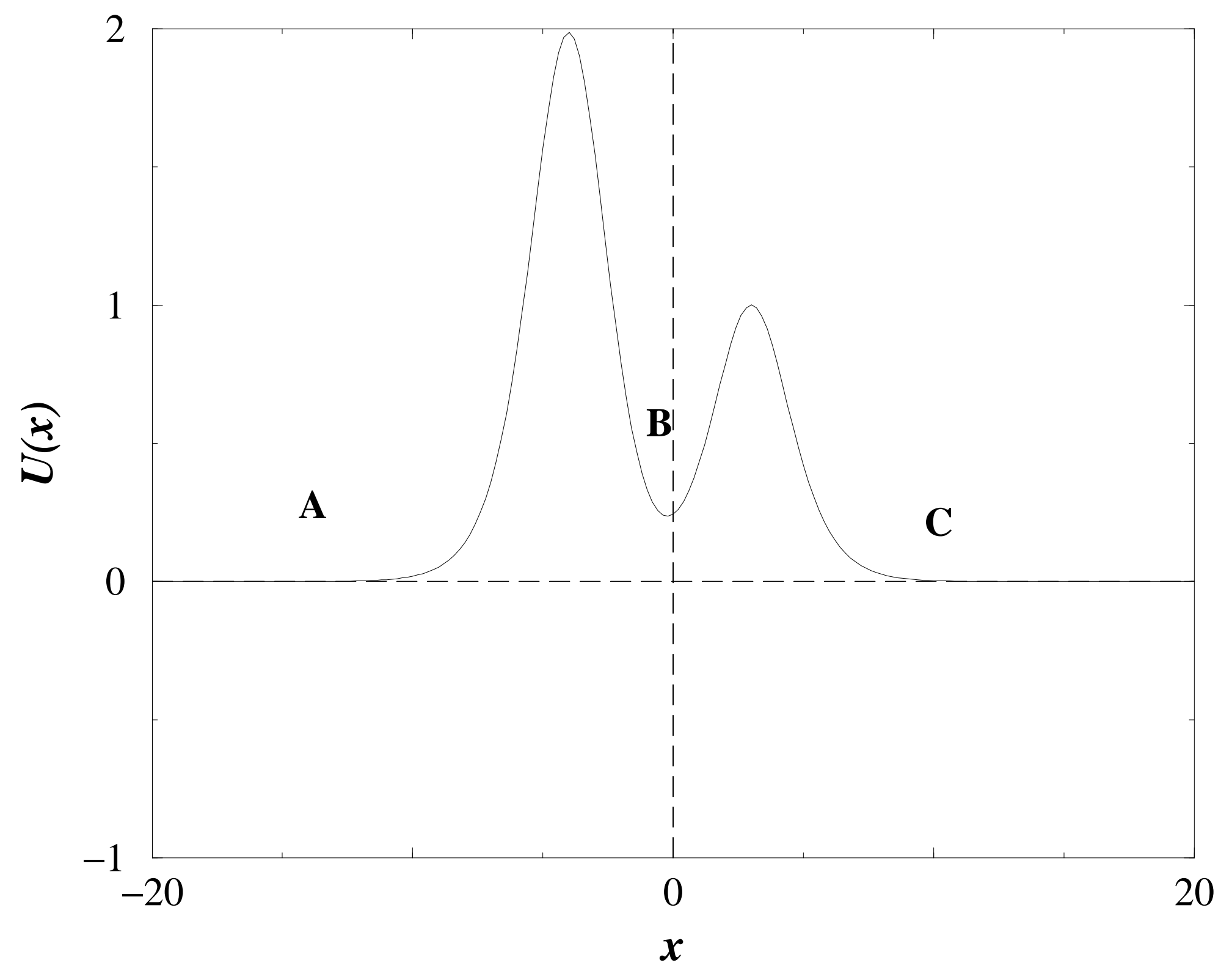

